\title{
An Investigation of Apoptosis in Androgenetic Alopecia
}

\author{
Michael B. Morgan ${ }^{1}$ and Paul Rose ${ }^{2}$ \\ ${ }^{1}$ Department of Pathology and 2 Department of Dermatology, College of Medicine, \\ University of South Florida,Tampa, Florida
}

\begin{abstract}
While the androgens, including dihydrotestosterone (DHT), have been implicated in the development of androgenetic alopecia (AGA), the exact mechanism by which they exert their effect(s) is unknown. Since apoptosis is an integral component of the normal cycling of human hair, we investigated individuals clinically affected by AGA to assess whether objective differences in the expression of apoptosisrelated immunohistochemical markers could be observed in scalp biopsies. Specimens from 16 alopecic male patients were stained with bcl-2 and the terminal deoxynucleotidyltransferase dUTP fluorescein nick end-labeling (TUNEL) method was used to assess apoptotic activity in affected and unaffected areas of the scalp. Immunoreactivity was analyzed by quantifying staining differences within the same individual. Sections from 3 human volunteers were used to establish the method validity. Significant differences in the bcl-2 staining index $(0.67$ versus $0.42, \mathrm{p}<0.05)$ and TUNEL expression $(5.7$ versus $10.2, \mathrm{p}<0.05)$ were observed between the areas of the scalp that were clinically affected (frontal) and unaffected (occipital) by AGA. The Gaussian distributions of bcl-2 and TUNEL staining suggest that a relatively uniform population of follicles exists at the frontal hairline and/or that synchrony of follicular cycling occurs in AGA. The apoptosis "hot spot" revealed by TUNEL staining in the bulge-isthmus region of the murine follicle is also identifiable in the human follicle. (received 1 July 2002; accepted 17 November 2002)
\end{abstract}

Keywords: alopecia, baldness, bcl-2, testosterone, TUNEL, apoptosis, hair follicle

\section{Introduction}

The fact that human males are prone to alopecia has been recognized for millennia. The nature of "male pattern" baldness was suspected centuries ago with Aristotle's observation that eunuchs never displayed the clinical condition now known as androgenetic alopecia (AGA) [1]. AGA results from recurrent premature termination of the growth (anagen) phase of the follicular cycle. Progressive anagen truncation results in miniaturized follicles, and ultimately, follicular loss associated with mild inflammation but no scarring.

In 1997, Sawaya and Price [2] demonstrated higher levels of androgen receptors in frontal scalp follicles than in occipital follicles, providing a micro-

Address correspondence to Michael B. Morgan, M.D., 16124 Chastain Road, Odessa, FL 33556, USA; tel 813971 0775; fax 813971 6675; e-mail mbkmmorgan@aol.com. anatomical explanation for the "classic" AGA pattern of frontal follicular miniaturization with occipital follicular preservation. These authors also noted regional differences in the expression of androgen converting enzymes $5-\alpha$ reductase I and II, and cytochrome P-450 aromatase on the scalp.

The classic pattern of AGA, while easily recognized and familiar to clinicians, has a few animal analogues: some chimpanzees and red uacaris and, chiefly, the stump-tailed macaque [1]. The latter primate shows a clinically distinct androgen-induced alopecia in both sexes, histologically resembling that seen in humans. Owing to limitations of the macaque as an experimental animal, researchers have avoided macaques in favor of the more easily handled murine model $[1,3]$.

Cycling of hair follicles in the mouse has been intensively investigated from the histological and molecular viewpoints $[3,4]$. It is clear that the 
mammalian hair follicle is an extremely complex organ that undergoes periods of growth (anagen), involution (catagen), and quiescence (telogen). Mediators suspected of dictating the cyclic action of the follicles in the murine model include dozens of biochemicals that have been studied in other cell signaling pathways, including those involved with the initiation of apoptosis or programmed cell death [3-12]. In 1997, Lindner et al [4] mapped the expression of biochemical mediators throughout the follicular cycle of mice, demonstrating the complex association of biochemical signaling with follicular phase changes.

In this study, we investigated apoptosis using 2 different staining techniques (ie, bcl-2, an inhibitor of apoptosis, and TUNEL, an indicator of apoptosis) (a) to see if the observations in the murine model hold true in human histologic sections, and (b) to determine whether there is a significant staining difference between the regions of the scalp that are affected and unaffected by androgenetic alopecia.

Scalp biopsies ( $4 \mathrm{~mm}$ punch) were obtained during 2001-2002 from 16 men undergoing hair transplantation (age 32-69 yr, mean $46 \mathrm{yr}$ ). The subjects manifested male pattern baldness classified as stage II-IV ( 4 at Stage II, 6 at Stage III, and 6 at stage IV) according to the Norwood classification scheme [5]. There were no indications of dermatological or systemic diseases that might have contributed to the development of alopecia in these subjects.

Two biopsies were obtained from each subject, one from the frontal scalp at the apex of a coronal plane through the ear tragi, and the other from the normally appearing occipital scalp. The biopsies specimens were processed in the usual manner and embedded in paraffin after being cut both longitudinally and in cross-section. At least 25 sections, cut at $5 \mu \mathrm{m}$ intervals, were mounted on glass slides in numerical sequence. Every fifth slide was stained with hematoxylin and eosin $(\mathrm{H} \& \mathrm{E})$; the remaining slides were saved for immunohistochemical studies. Unstained sections were selected to demonstrate the dermal papilla (DP) for bcl-2 immunohistochemical staining and the infundibulum for TUNEL staining (as described below). The identical procedure was performed on 3 human volunteers (men, ages 36, 47, and $52 \mathrm{yr}$ ) with no alopecia, who served as controls.

The cases were assigned random numbers and independently evaluated by a single pathologist (MBM) who was blinded in respect to subject identity and anatomic site. Total numbers of follicles were enumerated, including the miniaturized type and the terminal type, as well as the percentages of anagen, catagen, and telogen types. A ratio of such types within each region was calculated for each patient. The number of cells in the dermal papillae that stained positively for bcl-2 was quantified at $400 \mathrm{x}$ magnification. TUNEL staining was evaluated qualitatively (present or absent) with attention to microanatomical location. Staining indices (ie, the numbers of cells that stained positively for bcl- 2 and the numbers of nuclei with TUNEL, divided by the total number of cells or nuclei visible) were calculated for each visualized follicle.

The individual follicle indices were averaged to arrive at a single frontal staining index (FSI) and occipital staining index (OSI) for each subject studied. Twelve follicles were examined at high power magnification $(400 \mathrm{x})$ for each sample, with 20 nuclei or cells analyzed per follicle. The F-test was used to assess the variances between the two data sets; the significance of differences between means was computed by the paired-sample t-test.

The TUNEL procedure was performed using the "ApopTag Plus In Situ Apoptosis Detection Kit" (Oncor, Inc., Gaithersburg, MD), according to the manufacturer's instructions with the following amendments: (a) protein digestion was performed with the Oncor protein digesting enzyme at room temperature for $10 \mathrm{~min}$; (b) the $\mathrm{DAB}$ reagent was supplied by Sigma Chemical Co. (St. Louis, MO); and (c) counterstaining was performed with Mayer's hematoxylin rather than methyl green. Positive controls consisted of tonsillar tissue; negative controls consisted of the identical tissue with omission of the secondary antibody from the standard staining procedure.

For bcl-2 staining, microwave antigen retrieval was performed by treatment in citrate buffer $(\mathrm{pH}$ 6.0) for 20 min. Bcl-2 antibody (Ventana, Tucson, AZ, prediluted) was used with an automated stainer (Ventana, Inc., Tucson, AZ), with hematoxylin 


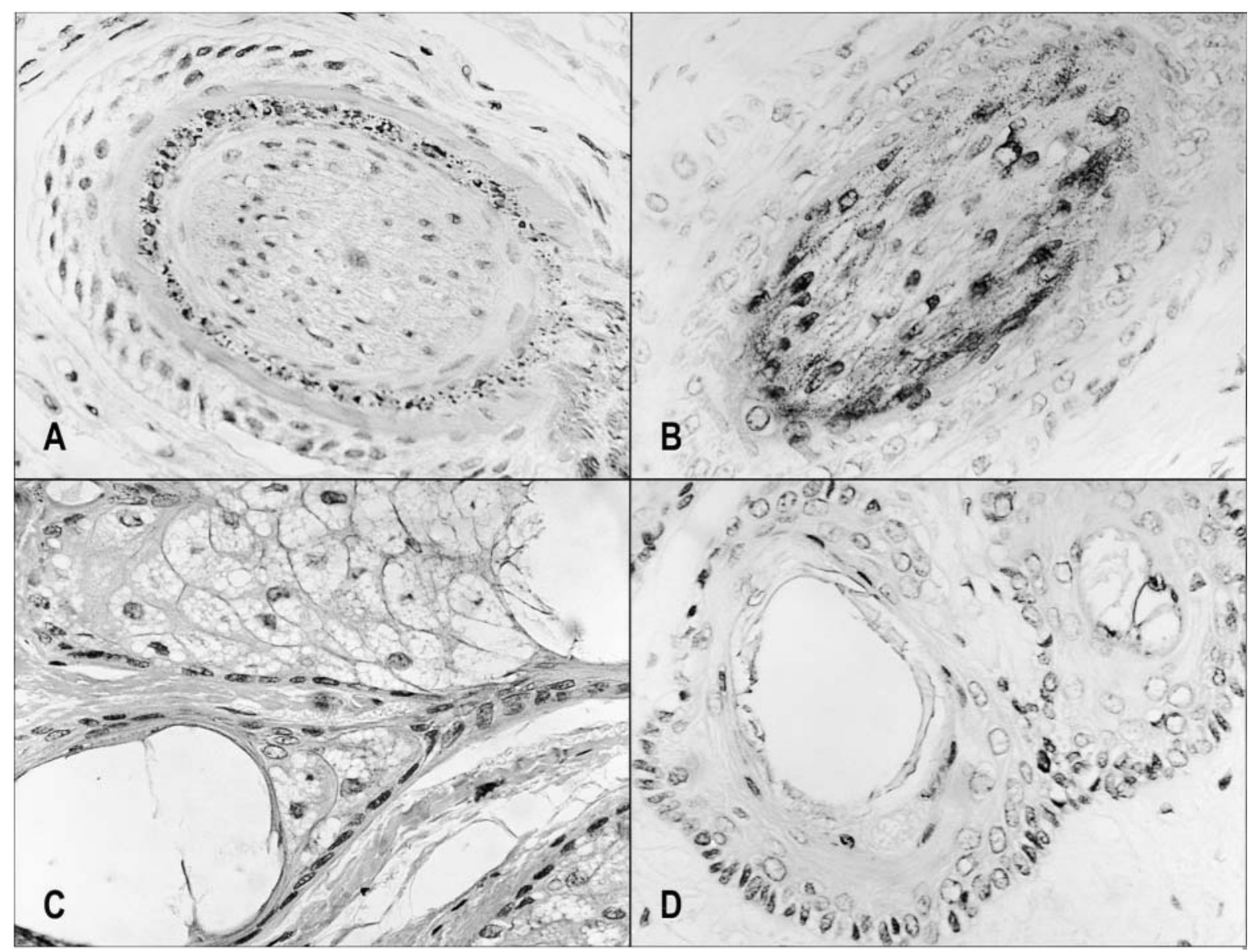

Fig. 1. Immunohistochemical staining of hair follicles from male patients with androgenetic alopecia (magnification $\mathrm{x} 100$ ). Panel A: bcl-2 immunostaining of a follicle from the occipital scalp. Panel B: bcl-2 immunostaining of a follicle from the frontal scalp. Panel C: TUNEL stain of a follicle from the frontal scalp. Panel D: TUNEL stain of a follicle from the occipital scalp.

counterstaining. Positive and negative external controls consisted of tonsillar tissue, as noted above. Positive internal controls consisted of eccrine ductal epithelium and dermal lymphocytes, both of which typically stain positively with bcl-2 antibody.

\section{Results}

All 16 patients were suitable for study, comprising 32 samples (16 frontal, 16 occipital). Representative $H \& E$ stained sections of the frontal scalp specimens showed the changes of androgenetic alopecia, with miniaturized follicles and a sparse peribulbar chronic inflammatory infiltrate. Specimens from the occipital scalp showed normal terminal follicles with rare miniaturized follicles. The majority of follicles from the frontal scalp were miniaturized, with an average of 10.1 ( $S D \pm 0.9)$ miniature follicles observed per 12 follicles examined (83\%). The occipital specimens yielded an average of 2.1 (SD \pm 0.4 ) miniature follicles per 12 examined (17\%). The majority of the follicles within the frontal scalp were anagen $(9.6 \pm 0.5)$ with the remainder in catagen $(0.6 \pm 0.8)$, or telogen $(2.1 \pm 0.7)$ per 12 examined. The follicles from occipital scalp showed a similar distribution of anagen (9.8 \pm 0.5$)$, catagen ( $0.3 \pm$ $0.5)$, and telogen (1.8 \pm 0.4$)$ per 12 examined.

The quality of the bcl-2 stain did not differ from that seen in patient biopsies and controls, each showing cytoplasmic immunoreactivity. The quality 
of the bcl-2 staining was similar among miniaturized and terminal follicles, and showed qualitatively less intense staining in the catagen and telogen follicles. $\mathrm{Bcl}-2$ staining was demonstrated in all anagen follicles examined. The average anagen staining index for the frontal scalp (FSI) was 67\%, consisting mostly of miniaturized follicles. The average anagen staining index for the occipital scalp (OSI) was $42 \%$, consisting mostly of terminal follicles (Table 1 ).

The FSI for bcl-2 staining in the controls was $41 \%, 46 \%$, and $37 \%$ (average $=41 \%$, Table 1 ). The OSI for the control group was 33\%, 43\%, and 48 (average $=43 \%$, Table 1; Fig. 1, panels A and B). The F statistic of the patients' FSI versus their OSI was 1.21 , indicating no significant differences in the variances of the 2 groups of biopsy staining patterns. In patients with Norwood classification II-IV the F statistics within frontal and occipital scalp were 1.12 and 1.36 respectively, indicating no significant differences in the variances of the 2 groups. The paired t-test revealed a t statistic of $2.20(\mathrm{p}<0.05)$.
FSI results revealed a non-skewed distribution of indices in the studied subjects. The OSI results for bcl-2 staining were negatively skewed, indicating that the majority of follicles from the posterior scalp displayed a smaller proportion of staining cells, compared to the OSI mean. Dermal eccrine duct epithelium and lymphocytes stained with the bcl-2 antibody in all slides examined.

The TUNEL staining in all patients was localized to the central portions of sebaceous glands and the immediately adjacent hair follicle. The quality of the staining was nuclear, and did not differ from that observed in normal scalp controls. There was no difference in the quality of staining between miniaturized and terminal follicles. Increased TUNEL labeling was observed within the catagen terminal and miniaturized follicles of the frontal and occipital follicles of controls and study patients. Anagen follicles from all categories showed an intermediate staining intensity and telogen follicles showed nearly absent staining. Within the follicles,

Table 1. Labelling indices (expressed as percentages) for bcl-2 and TUNEL staining of hair follicles from the frontal scalp and the occipital scalp of 16 male patients with androgenetic alopecia and from 3 control men.

\begin{tabular}{|c|c|c|c|c|}
\hline \multirow{2}{*}{$\begin{array}{c}\text { Category of } \\
\text { subject }\end{array}$} & \multicolumn{2}{|c|}{ Frontal scalp specimens } & \multicolumn{2}{|c|}{ Occipital scalp specimens } \\
\hline & bcl-2 (\%) & TUNEL (\%) & bcl-2 (\%) & TUNEL (\%) \\
\hline \multicolumn{5}{|c|}{ Androgenetic alopecia } \\
\hline 1 & 56 & 5.1 & 40 & 9.9 \\
\hline 2 & 71 & 5.9 & 49 & 10.8 \\
\hline 3 & 74 & 5.0 & 42 & 9.7 \\
\hline 4 & 63 & 5.9 & 50 & 9.9 \\
\hline 5 & 60 & 6.8 & 39 & 10.4 \\
\hline 6 & 74 & 6.0 & 45 & 10.0 \\
\hline 7 & 63 & 5.8 & 44 & 11.1 \\
\hline 8 & 64 & 5.8 & 42 & 9.6 \\
\hline 9 & 70 & 4.9 & 41 & 9.3 \\
\hline 10 & 71 & 6.8 & 48 & 9.8 \\
\hline 11 & 76 & 5.6 & 39 & 10.8 \\
\hline 12 & 51 & 5.9 & 43 & 10.6 \\
\hline 13 & 69 & 6.2 & 36 & 10.9 \\
\hline 14 & 70 & 4.9 & 34 & 11.1 \\
\hline 15 & 59 & 4.8 & 41 & 9.8 \\
\hline 16 & 73 & 5.6 & 39 & 9.6 \\
\hline Average & 67 & 5.7 & 42 & 10.2 \\
\hline \multicolumn{5}{|l|}{ Control men } \\
\hline 1 & 41 & 9.6 & 33 & 10.0 \\
\hline 2 & 46 & 9.9 & 43 & 10.8 \\
\hline 3 & 37 & 10.4 & 48 & 9.4 \\
\hline
\end{tabular}


nuclear TUNEL staining in cells of the inner root sheath localized to the bulge-isthmus region of the follicle was observed.

The average anagen staining index (ie, the average percentage of cells stained for TUNEL per follicle) for the FSI was $5.7 \%$ and for the OSI was 10.2\% (Table 1; Fig. 1, panels C and D). For TUNEL staining in controls, the FSI was $9.8 \%$ and the OSI was $10.1 \%$. The F statistic of the FSI versus the OSI was 1.01, and within all Norwood groups 1.41 and 1.59 respectively. The paired t-test revealed a $t$ statistic of $3.01(p<0.05)$ for TUNEL FSI vs OSI.

\section{Discussion}

The finding of bcl-2 staining in all follicles examined is consistent with observations of Lindner et al [4]. They found that bcl-2 staining was evident throughout catagen and anagen in cycling dermal papillae. Not surprisingly, the staining indices in our study were different in the frontal (FSI) vs the occipital (OSI) follicles. That the FSI was consistently higher than the OSI was unexpected. Since bcl-2 is regarded as an "anti-apoptosis" marker, the fact that a larger proportion of frontally located dermal papilla (DP) cells (in a clinically active area of androgenetic alopecia) would be positive for this marker seems counterintuitive.

One possible explanation is that the vellus follicles identified in the frontal biopsies may represent a relatively resistant population that has not yet been "earmarked" for involution. This "survival" hypothesis suggests that these few remaining follicles are either equipped to resist involution, or have not yet been irresistibly influenced by biochemical signals that would eventually result in their disappearance. Thus, they may represent a relatively pure population of follicles (at least temporarily) resistant to apoptosis.

That the frontal follicles may represent a "pure" population is further suggested by the evenly distributed FSI values. This distribution indicates that the majority of frontal dermal papillae manifest a staining index clustered around the mean, with relatively few papillae exhibiting markedly hypo- or hyper-active staining. This finding is particularly interesting since the distribution of occipital follicle staining indices is unevenly distributed. This negatively skewed OSI distribution indicates that the great majority of occipital dermal papillae manifest a decreased staining pattern for bcl-2, with a minority exhibiting relatively hyperactive staining. This skewed pattern suggests either an asynchronous cycling follicular population, or two (or more) populations of follicles, each demonstrating varying degrees of bcl-2 staining. Observations in clinical and animal models favor the former hypothesis.

Follicular cycling involves a complex and coordinated interaction between the bulge stem cell region, the dermal papilla, and the matrix cells. It has been postulated that the dermal papilla orchestrates stem cell activation "upstream" to the events of terminal differentiation and apoptosis in the bulge and matrix areas $[13,14]$. In this model, the papilla, as master controller of an apoptosismediated follicular cycle, could theoretically be protected from the flurry of nearby follicular involution by bcl-2 expression.

Our results show that the degree of expression of bcl-2 in frontal follicles relative to occipital follicles is increased in individuals with clinically evident AGA. As our study did not include a large number of biopsies from non-AGA affected individuals, it is unclear what level of bcl-2 expression would be considered "normal" in human frontal follicles. Whether the presence of bcl-2 in frontal follicles of patients with AGA is a pathological expression or overexpression is unanswered. If subsequent studies reveal a pathological overexpression in frontal AGA follicles, one could speculate that persistently elevated bcl-2 expression could lead to immortalization of the dermal papilla. This could potentially exhaust the follicular stem-cell pool, resulting in progressively smaller follicles.

Our study set is too small to reach meaningful associations between bcl-2 staining indices and follicular stage, although others have attempted this in mouse studies [4,9]. When bcl-2 overexpression was studied in outer root sheath keratinocytes in transgenic mice treated with cyclophosphamide, increased catagen induction was observed $[15,16]$. Specific catagen-promoting factors were not identified, however. As one of the regulators of the 
hair cycle [3] and a promoter of catagen, FGF-5 was suggested, though not proven [16].

The results obtained with TUNEL staining were anatomically different with a higher rate of apoptosis obtained in the occipital region. Given the antithetical relationship between bcl-2 expression and apoptosis as assessed by the TUNEL method, it would be expected that the apoptotic rate would be higher in the occipital region and lend credence to the bcl-2 findings. The intranuclear staining observed in the bulge-isthmus region of a follicle (Fig. 1, panel D) correlates well with one of the four TUNEL-associated apoptotic "hot spots" described in the murine model [4].

In summary, our findings suggest that the propensity to inherit AGA may represent increased susceptibility to mediators of bcl-2 expression or qualitatively abnormal bcl-2 responsive elements (receptors) within the DP of the alopecia-prone frontal areas of the scalp. Further studies of AGA are needed to assess the expression of IGF-1, BAD, FGF-5, TGF- $\beta$, and possibly other genes in the bcl-2 family that have been implicated in murine follicular cycling. We plan future investigations that will focus on the dermal papilla, its important role as a coordinator of hair follicle cycling, and the alterations responsible for androgenetic alopecia.

\section{References}

1. Olsen, EA: Disorders of Hair Growth: Diagnosis and Treatment, McGraw-Hill, New York, 1994.

2. Sawaya ME, Price VH. Different levels of 5- $\alpha$ reductase type I and II, aromatase, and androgen receptor in hair follicles of women and men with androgenetic alopecia. J Invest Dermatol 1997;109:296-300.
3. Stenn KS, Combates NJ, Eilertsen KJ, et al. Hair follicle growth controls. Dermatol Clin 1996;14:543-557.

4. Lindner G, Botchkarev VA, Botchkareva NV, Ling G, van der Veen C, Paus R. Analysis of apoptosis during hair follicle regression (catagen). Am J Pathol 1997;151:16011617.

5. Olsen EA, Weiner MS, Delong ER, Pinnell SR. Topical minoxidil in early male pattern baldness. J Am Acad Dermatol 1985;13:185-192.

6. Wyllie AH, Kerr JFR, Currie AR. Cell death: the significance of apoptosis. Int Rev Cytol 1980;68:251-306.

7. Polakowska RR, Piacentini M, Bartlett R, Goldsmith A, Haake AR. Apoptosis in human skin development: morphogenesis, periderm, and stem cells. Dev Dyn 1994; 199:176-188.

8. Tamada Y, Takama H, Kitamura T, et al. Identification of programmed cell death in normal human skin tissues by using specific labeling of fragmented DNA. Br J Dermatol 1994;131:521-524.

9. Stenn KS, Lawrence L, Veis D, Korsmeyer S, Seiberg M. Expression of the bcl-2 protooncogene in the cycling adult mouse hair follicle. J Invest Dermatol 1994;103:107-111.

10. Steller H. Mechanisms and genes of cellular suicide. Science 1995;267:1445-1449.

11. Jacobson MD, Burne JF, King MP, et al. Bcl-2 blocks apoptosis in cells lacking mitochondrial DNA. Nature 1993;361:365-369.

12. Thompson CB. Apoptosis in the pathogenesis and treatment of disease. Science 1995;267:1456-1462.

13. Soma T, Ogo M, Suzuki J, Takahashi T, Hibino T. Analysis of apoptotic cell death in human hair follicles in vivo and in vitro. J Invest Dermatol 1998;111:948-954.

14. Cotsarelis G, Sun T-T, Lavker RM. Label-retaining cells reside in the bulge area of pilosebaceous unit: implications for follicular stem cells, hair cycle, and skin carcinogenesis. Cell 1990;61:1329-1337.

15. Muller-Rover S, Rossiter H, Lindner G, et al. Hair follicle apoptosis and bcl-2. J Invest Dermatol Sym Proc 1999; 4:272-277.

16. Muller-Rover S, Rossiter H, Paus R, et al. Overexpression of bcl-2 protects from ultraviolet $\mathrm{B}$-induced apoptosis but promotes hair follicle regression and chemotherapyinduced alopecia. Am J Pathol 2000;156:1395-1405. 